\title{
A Computer-Assisted ACL Reconstruction System Assessment of Two Techniques of Graft Positioning in ACL Reconstruction
}

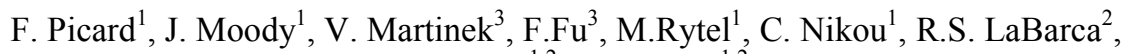 \\ B. Jaramaz ${ }^{1,2}$, A.DiGioia ${ }^{1,2}$.
}

Centers for Medical Robotics and Computer-Assisted Surgery,

${ }^{1}$ UPMC Shadyside Hospital, Pittsburgh, PA and

${ }^{2}$ Robotics Institute, Carnegie Mellon University, Pittsburgh, PA.

${ }^{3}$ Department of Orthopaedic Surgery, University of Pittsburgh, Pittsburgh, PA.

\begin{abstract}
KneeNav ${ }^{\mathrm{TM}} \mathrm{ACL}$, a CT-based surgical navigation system, is used to demonstrate two fundamental requirements for a practical computer-assisted surgical device: 1) it can guide the surgeon with the accuracy required for successful graft placement, and 2) this guidance can be achieved through a simple and intuitive interface. A series of ACL reconstruction preparation procedures were performed on foambone models using traditional arthroscopic techniques. Half of the procedures were done using conventional techniques, the other half were performed with $\mathrm{KneeNav}^{\mathrm{TM}} \mathrm{ACL}$ guidance. Resulting tunnel and femoral graft site locations were measured via radiographic and computer means, and the two techniques are compared. Ease of system use is also assessed.
\end{abstract}

Keywords: Computer-assisted surgery, navigation systems, ACL surgery.

\section{Introduction}

The outcomes following treatment of anterior cruciate ligament injuries have been shown to be highly dependent on the surgical technique employed. Two main clinical challenges faced by surgeons are graft placement and tensioning. Outcomes following ACL reconstruction depend on the selection of the graft position and the initial graft tension. Optimizing these two factors is expected to improve outcomes and reduce long-term complications $[1,2]$. Although ACL reconstruction is a routine procedure, accurate ACL graft placement remains a difficult task even using current arthroscopic techniques. Alternatively, a computer-guided surgical system could be used to intraoperatively locate ideal graft placement sites.

The aim of this study was to evaluate two techniques for guiding holes used for graft attach during ACL reconstruction. These were a traditional "free hand" method using standard guide-pin tools, and a computer-assisted surgical guidance system that augments the standard tool set. The specific goals were:

- To assess and compare the accuracy, reliability and repeatability of the two ACL graft positioning techniques.

- To test whether the use of a computer-assisted surgical guidance system can enhance a surgeon's ability to consistently achieve a predetermined (i.e. target) plan. 


\section{Material and Methods}

\subsection{Personnel, Equipment \& Materiel Requirements:}

The following list summarizes the main requirements for the core experiments.

- Two surgeons experienced in ACL reconstruction, with little previous exposure to computer-assisted surgical guidance systems.

- 21 sawbones knee models per surgeon. These are custom knee models complete with meniscus, collateral ligaments and PCL. All are fully enclosed in a simulated capsule of light-impervious elastic material.

- An arthroscopic station suitable for ACL reconstruction surgery included 1) a thirty degree optical device, 2) a camera plugged onto the optical device, 3) central processing unit, 4) light cable, 5) monitor, and 6) a VCR to record the experimental data.

- A traditional ACL tool set (Paramax ${ }^{\mathrm{TM}}$ ACL guide system) and a drill.

- Computer-assisted guidance system $\left(\mathrm{KneeNav}^{\mathrm{TM}}\right)$, including trackers to be attached to the femur, tibia, and the Paramax ${ }^{\mathrm{TM}}$ cruciate guide assembly (guide pin).

\subsection{Design and Execution of the Experiment}

\subsubsection{General}

During the experiment two surgeons independently prepared ACL tunnels on sets of sawbones knee models. Each surgeon used both traditional and computer-guided methods to attempt to place the femoral and tibial tunnels in a pre-determined orientation and location. Postoperatively, the tunnel positions and orientations were measured. In addition to direct physical measurements, computer and radiographic analysis techniques were used.

Foambones knee models were used in the experiment since they are readily available, anatomically accurate, and (to within manufacturing tolerances) physically identical. Thus, meaningful results can be obtained by comparing follow-up measurements from all sawbones test specimens. Although not truly representative of an actual surgical environment, careful test site preparation and the use of sawbones knee models provided sufficiently realistic OR conditions for the purposes of this study.

\subsubsection{Preparation:}

Several days before the experiment sessions each surgeon was given a sawbone knee model from which the simulated capsule was removed. On this model the surgeon indicated the exact locations of the desired entry and exit holes for ACL tunnels. This reference knee then became, in effect, that surgeon's preoperative plan for the surgical experiment. The participating surgeons used their specific reference model throughout the experiment. This gave each surgeon the advantage of working within their personal preference, but limits how the experimental data can be related across all participants.

Each reference knee's hole locations were marked with radio-opaque fiducials. The model was CT scanned using the following protocol. 
All slices were $1 \mathrm{~mm}$ thick. Inter-slice density varies depending on the location in the model:• $1 \mathrm{~mm}$ spacing was used in critical areas, e.g., areas used for registration and areas containing fiducials. $\bullet 3 \mathrm{~mm}$ spacing is used in between high-density areas, and in other areas of anatomic interest. $\bullet 10 \mathrm{~mm}$ spacing is used in all other areas (most proximal and most distal).

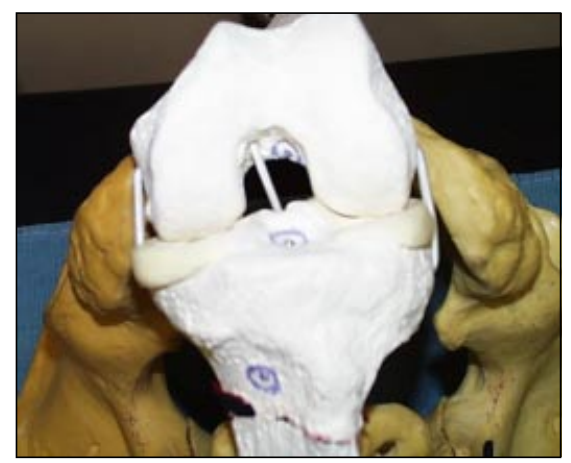

Figure 1: Sawbones model with radio-opaque fiducials.

Computer surface models were generated from the CT scan. These models were used intraoperatively with the computer-assisted surgical guidance system as well as in the post-operative analysis.

\subsubsection{Randomized Procedure}

The study was a randomized prospective parallel investigation comparing traditional and computer-assisted guided approaches to ACL reconstruction. Beforehand, two groups of twenty identical knee models were selected. Models within these groups were numbered respectively from 1 to 20 for the first surgeon and from 21 to 40 for the second surgeon. For each surgeon a set of 20 envelopes was prepared indicating whether the "Traditional technique" or "Computer-Guided technique" was to be performed. The 20 envelopes were combined and thoroughly mixed, thus randomizing the subsequent selection order.

During the experimental sessions the knee models were processed in numerical order. Once a model was set up, an envelope was selected and the indicated technique was used to perform the ACL tunnel placement on that model.

\subsubsection{Surgical Procedure}

Each surgery was performed on a new sawbones model. Each model was placed in an operative posture and secured in a fixture designed to permit the surgeon the use of a standard surgical approach. The models had fully intact capsules to minimize any external visual cues for tunnel placement.

In the traditional technique, the tibial guide-pin was arthroscopically positioned. A $10 \mathrm{~mm}$ bit was then used to over-drill the tibia tunnel along the guide-pin. Next, the guide-pin was used through the tibial tunnel to place the femoral tunnel entry point. The pin was drilled through the femur. The femoral tunnel was not over-drilled. 


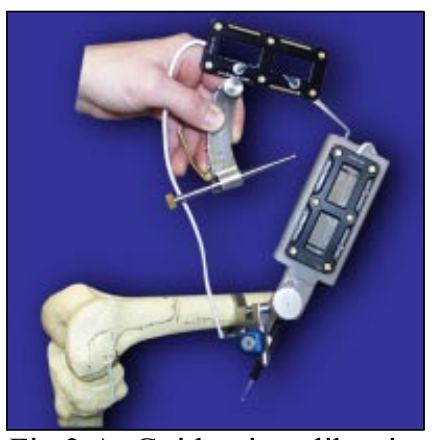

Fig 2 A: Guide-pin calibration

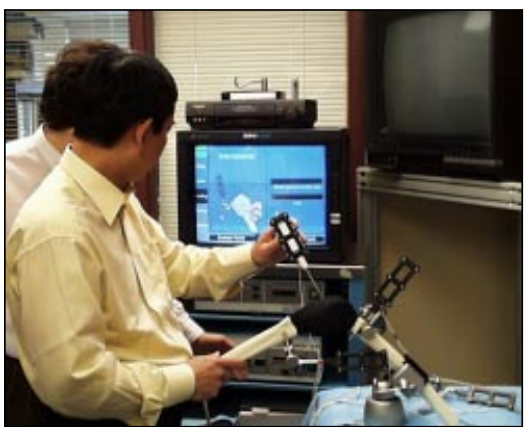

Fig 2 B: Data collection

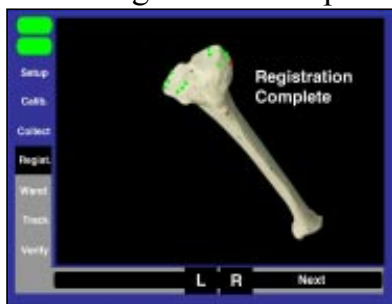

Fig 2 C: Registration.

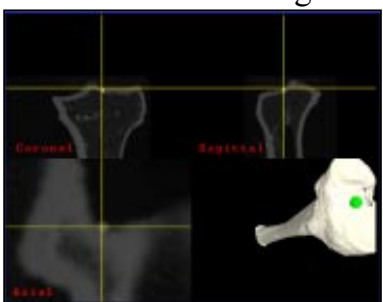

Fig 2 D: Verification

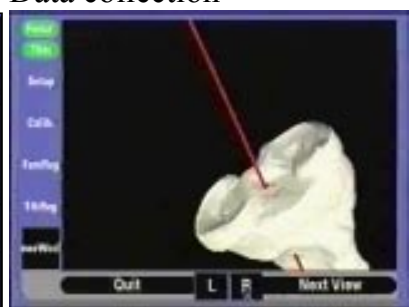

Fig 2 E: Guide-pin orientation

In the computer-assisted technique the following steps were performed prior to the normal surgical flow: device calibration, data registration and verification. Then, using the calibrated guide-pin, the surgeon placed the tibial and femoral tunnels as above, but under computer guidance. The arthroscope was not used during the computer technique.

A gray line (fig 2E) represented the guide-pin and gray dot marked the predetermined ACL sites (surgical goal). Three different knee views (tibial superior view, femoral inferior view and tibio-femoral sagittal view) were continuously displayed on the monitor and could be selected by the surgeon using a foot pedal control.

The surgery then resumed in a traditional manner. No notchplasty was performed.

\subsubsection{Evaluation Protocol}

For follow-up analysis each model was carefully reregistered, and the final position of the femoral and tibial holes were recorded.

The main assessment criterion was the radiological measurement of the femoral and tibial hole positioning. The following parameters were measured from X-rays for each of the foambone models by two observers. (Figure 3): FSP $=$ Femoral hole sagittal positioning; FCP = Femoral hole coronal positioning; TSP = tibia hole sagittal positioning; and TCP $=$ Tibial hole coronal positioning.

In order to standardize the x-ray measurements a casting was prepared to secure the identical sawbones models in exactly the same film orientation. Each model was filmed in the AP and lateral views. 


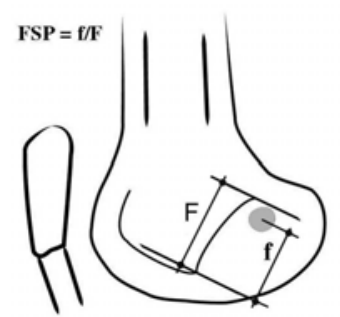

$$
\begin{gathered}
\mathrm{FSP}=\text { femoral } \\
\text { hole sagittal } \\
\text { position }
\end{gathered}
$$

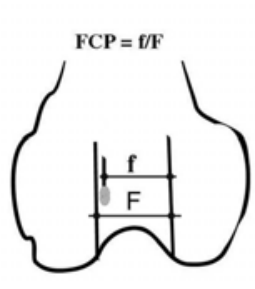
$\mathrm{FCP}=$ femoral hole frontal position.
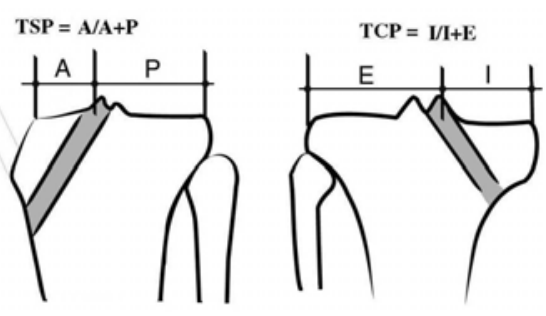

TSP $=$ Tibial hole $\quad$ TCP $=$ tibial hole sagittal position. frontal position.

Figure 3: Technique of X-ray measurements.

For the follow up measurements the knee model was equipped with rigid bodies (one each for the tibia and femur) then registered in the normal "Nav" fashion. Good registration was verified. A centering rod was inserted through the tibial tunnel so that each end of the rod could be easily accessed, as illustrated in Figure 4. The ball probe tip was inserted into the distal detent, and the position was measured with respect to the tibia. The ball probe was moved to the proximal detent and a second measurement was recorded. For femoral measurements the ball probe was placed directly on each end of the femoral guide-pin tunnel. The points were collected with respect to the femoral tracker. The ball probe self-centered to the tunnel ends for most knee models. On models with oblique femoral tunnels care had to be taken to center the probe tip. Post processing software computed the intersection of the tunnel axes with the bone surface model.
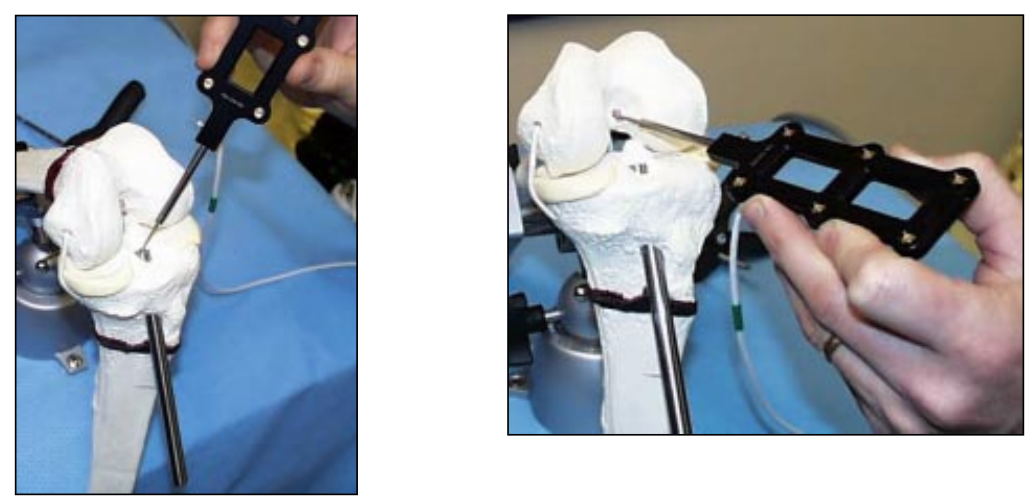

Figure 4: Computer-assisted measurement technique. 


\section{Results}

Table I: Measurements of the bone holes positioning.

\begin{tabular}{|c|c|c|c|c|c|}
\hline \multirow{2}{*}{$\begin{array}{c}\text { Hole } \\
\text { position }\end{array}$} & \multirow{2}{*}{$\begin{array}{l}\text { Measurement } \\
\text { technique }\end{array}$} & \multicolumn{2}{|c|}{ Surgeon number one } & \multicolumn{2}{|c|}{ Surgeon number two } \\
\hline & & Scope & KneeNav & Scope & KneeNav \\
\hline \multirow[b]{2}{*}{$\begin{array}{l}\text { FCP } \\
\text { Coronal } \\
\text { femoral } \\
\text { hole } \\
\text { position. }\end{array}$} & \multirow{2}{*}{$\begin{array}{c}\text { X-ray } \\
\text { measurement }\end{array}$} & $75.7+/-6.7$ & $70.7+/-8.9$ & $79.8+/-8.7$ & $90.1+/-4.9$ \\
\hline & & \multicolumn{2}{|c|}{$\begin{array}{c}\text { Goal }=70.1 \\
\mathrm{p}=0.08\end{array}$} & \multicolumn{2}{|c|}{$\begin{array}{c}\text { Goal }=\mathbf{1 0 0} \\
\mathrm{p}=0.01\end{array}$} \\
\hline \multirow{2}{*}{$\begin{array}{c}\text { FSP } \\
\text { Sagittal } \\
\text { femoral } \\
\text { hole } \\
\text { position }\end{array}$} & \multirow{2}{*}{$\begin{array}{c}\text { X-ray } \\
\text { measurement }\end{array}$} & $67.9+/-3.37$ & $68+/-7.13$ & $66.9+/-4.41$ & $82.5+/-8.4$ \\
\hline & & \multicolumn{2}{|c|}{$\begin{array}{c}\text { Goal }=\mathbf{7 2} \\
\mathrm{p}=0.94\end{array}$} & \multicolumn{2}{|c|}{$\begin{array}{c}\text { Goal }=\mathbf{8 3 . 4} \\
\mathrm{p}=0.71\end{array}$} \\
\hline \multirow{2}{*}{$\begin{array}{c}\text { TCP } \\
\text { Coronal } \\
\text { tibial hole } \\
\text { position. }\end{array}$} & \multirow{2}{*}{$\begin{array}{c}\text { X-ray } \\
\text { measurement }\end{array}$} & $47.5+/-1.52$ & $50.3+/-1.48$ & $48+/-2.03$ & $51.9+/-1.93$ \\
\hline & & \multicolumn{2}{|c|}{$\begin{array}{c}\text { Goal }=\mathbf{5 2 . 2} \\
\mathrm{p}=0.98\end{array}$} & \multicolumn{2}{|c|}{$\begin{array}{c}\text { Goal }=\mathbf{5 0 . 5} \\
\mathrm{p}=0.86\end{array}$} \\
\hline \multirow{2}{*}{$\begin{array}{c}\text { TSP } \\
\text { Sagittal } \\
\text { tibial hole } \\
\text { position }\end{array}$} & \multirow{2}{*}{$\begin{array}{c}\text { X-ray } \\
\text { measurement }\end{array}$} & $41.1+/-2.04$ & $35.1+/-4.5$ & $33.5+/-4.21$ & $35.5+/-3.04$ \\
\hline & & \multicolumn{2}{|c|}{$\begin{array}{c}\text { Goal }=\mathbf{3 9 . 1} \\
\mathrm{p}=0.02\end{array}$} & \multicolumn{2}{|c|}{$\begin{array}{c}\text { Goal }=\mathbf{3 6} \\
\mathrm{p}=0.08\end{array}$} \\
\hline
\end{tabular}

No statistical differences between inter-observer radiological measurements have been found.

Table II: Comparison between Scope

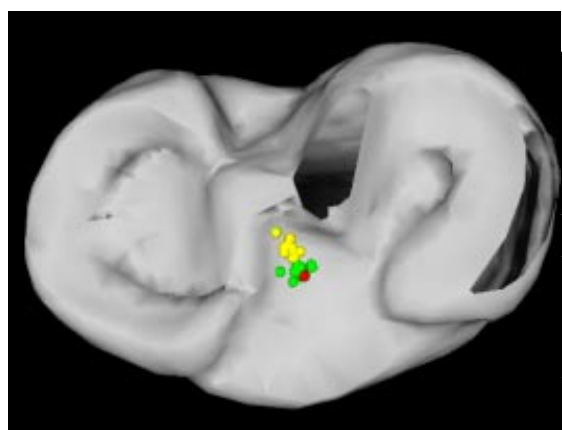
and KneeNav techniques $(\mathrm{p}=0.008)$

\begin{tabular}{|c|c|c|}
\hline $\mathrm{N}=40$ & $\begin{array}{c}\text { Scope } \\
\text { Technique } \\
(\mathrm{N}=20)\end{array}$ & $\begin{array}{c}\text { KneeNav- } \\
\text { ACL } \\
(\mathrm{N}=20)\end{array}$ \\
\hline Min & $4.07 \mathrm{~mm}$ & $2.05 \mathrm{~mm}$ \\
\hline Max & $10.01 \mathrm{~mm}$ & $15.08 \mathrm{~mm}$ \\
\hline $\begin{array}{c}\text { Average } \\
\text { distance to } \\
\text { the goal }\end{array}$ & $6.86 \mathrm{~mm}$ & $4.6 \mathrm{~mm}$ \\
\hline $\begin{array}{c}\text { Standard } \\
\text { Deviation }\end{array}$ & $1.69 \mathrm{~mm}$ & $2.7 \mathrm{~mm}$ \\
\hline
\end{tabular}

Figure 5: Target in black, computerDeviation assisted technique in gray and traditional technique in white. 
In order to evaluate the learning curve for surgeons using computer-assisted system, we normalized each measurement to the target values (T1, T2, T3, and T4). For each experiment appraisal we used four views: one coronal and one lateral/ sagittal view for each the femoral and the tibial holes $\left(X_{1 n}, X_{2 n}, X_{3 n}\right.$ and $\left.X_{4 n}\right)$ respectively. Figures below show additional errors to the target values for both surgeons.

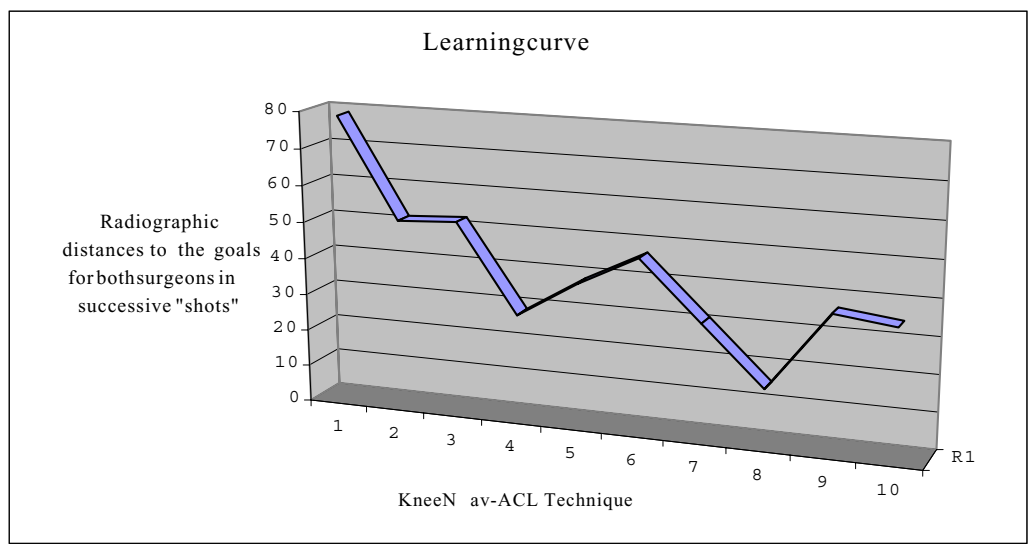

Figure 6: Learning curve. Cumulative errors to the goal during the computer-assisted surgery trial.

\section{Discussion}

The computer-assisted system enabled the surgeons to accurately place the tibial tunnel and femoral hole without direct visualization. Each surgeon placed the holes in accordance with his specific preoperative plan. Identical foambone knee models provided a common baseline for follow-up comparison.

However, because the surgeons did not reference a common plan the results had to be independently examined for radiographic appraisal. Neither surgeon's results showed a significant statistical difference between the traditional and computerassisted trials using the radiographic appraisal. The initial conclusion might be that the CAS system is comparable to the traditional tools in terms of accuracy in achieving goals. However, closer examination and computer-assisted measurements suggest another explanation.

As mentioned before, to prevent experimental bias we selected surgeons with no prior CAS system experience, but who did have extensive experience in ACL reconstruction surgery. A few iterations (about 4 or 5) with the CAS system were sufficient for the surgeons to become completely comfortable with the technology, demonstrating the ease of using CAS systems. However, since each surgeon performed a total of 10 tunnel placements under CAS guidance, only half can be 
considered to be outside of the learning curve. This resulting sample size is not sufficient for meaningful statistical analysis.

Even so, within these small sets we see a trend beginning to emerge. Beyond the initial learning curve the hole positions tend to be more tightly clustered around the planned locations as showed on figure 5. Once the surgeons became comfortable with the CAS system they were able to achieve the planned placement more accurately than with the traditional technique.

It is interesting to note that the tunnel sites preoperatively selected by each experienced surgeon differ appreciably $[1,5,6]$. For example, the femoral hole placement (coronal/sagittal) was 70/66 for surgeon 1, and 100/91 for surgeon 2 . This illustrates the difficulty in establishing a consensus regarding femoral tunnel position $[1,5,6]$, and provides a strong argument for a guidance system that allows surgeons to select tunnel sites. This also suggests a requirement for any surgical navigation system to support customizable planning parameters $[4,5,6]$. Moreover, the surgeon must be given the option to verify and/or change his plan intraoperatively. Such capability is not currently available in robotic-based surgical systems [7].

This study demonstrated the ease with which traditional surgical tools can be adapted for use in a computer-assisted surgical system. This is an important consideration for CAS systems to gain acceptance and make the transition to widespread practical use.

It was also noted that the surgical system could provide guidance that could not be fully exploited due to limitations in the traditional instrumentation. For example, in our study the tracked tibial guide pin could be oriented such that the tibial tunnel, when over-reamed, would provide optimal access to the intercondylar notch for the subsequent femoral tunnel placement. However, this optimal orientation could not always be maintained during tibial guide pin drilling because the oblique angle between the ACL guide cannula and the tibia prevented a stable purchase of the guide. A lesser angle had to be used to ensure stable guide positioning during pin drilling. Thus, although traditional tools can easily incorporated into computerassisted systems, instrumentation may have to be redesigned before surgeons can take full advantage of a computer-assisted system's capabilities.

The surgeons were exposed to new technical concepts related to CAS systems. Tracking issues, tool calibration, model registration, and operation of the userinterface were quickly grasped and posed no problem in the execution of the experiment [3, 4, 8]. Computer-Assisted Navigation Systems will provide viable alternatives to current surgical tools. However, CT scan is not a routine exam for ACL reconstruction and MRI models will probably more helpful in a near future. Regardless of the complexity of the underlying technology, a truly practical system must provide a simple and intuitive interface.

\section{Acknowledgements}

Chuck Cheetham from Linvatec Company, Bob Brown from Zimmer Randall Associate Inc. Company and Freddie Fu for additional support. 


\section{References}

1. Frank C.B, Jackson D.W., The Science of Reconstruction of the Anterior Cruciate Ligament. J Bone J.Surg 79-A, 1556-1576, 1997.

2. Fu F.H., Harner C.D., Johnson D.L. et al., Biomechanics of knee ligaments: Basic concepts and clinical application. Chapter 14, 137-148, 1994.

3. Sati M., Deguise J.A., Drouin G., Computer Assisted Knee Surgery: Diagnostics and planning of knee surgery. Computer Aided Surgery 2: 108-123, 1997.

4. Klos T.V.S., Banks A.Z., Banks S.A., et al., Computer and Radiographic Assisted Anterior Cruciate Ligament Reconstruction of the Knee. in Computer Assisted Orthopaedic Surgery L.P. Nolte and R.Gantz Hogrefe\& Huber Publishers.184-189, 1999.

5. Dessenne V., Lavallee S., Julliard R., et al., Computer Assisted Knee Anterior Cruciate Ligament Reconstruction: First Clinical Tests, Journal of Image Guided Surgery 1, 59-64, 1995.

6. Julliard R., Lavallee S., Dessenne V. Computer Assisted Reconstruction of the Anterior Cruciate Ligament. Clin.Orthop. Relat. Res. 354, 57-64, 1998.

7. Peterman J., Kober R., Heinze et al., Computer Assisted Planning and Robot Assisted Surgery in the Reconstruction of the Anterior Cruciate Ligament. Operative Techniques in Orthopaedics, 50-55, Vol 10, No 1 (January), 2000.

8. DiGioia A.M., Jaramaz B., Colgan B.D., Computer Assisted Orthopaedic Surgery, Clin. Orthop.Relat.Res., 354, 8-16, 1998.

9. Stulberg S.D, Picard F., Saragaglia D., Computer-Assisted Total Knee Arthroplasty. Operative Techniques in Orthopaedics, 25-39, Vol 10, No 1 (January), 2000. 